Original Paper http://ajol.info/index.php/ijbcs http://indexmedicus.afro.who.int

\title{
Contrôle de qualité des rideaux imprégnés à la bifenthrine en vue de leur utilisation et vulgarisation au Bénin
}

\author{
Anges YADOULETON ${ }^{1,2^{*}}$, Jean-RobertKLOTOE ${ }^{1}$, Ramziyath AGBANRIN ${ }^{2}$, \\ Fabrice AHISSOU ${ }^{2}$, Geraldo HOUNDETON ${ }^{2}$, Roland TOSSOU ${ }^{2}$, Achaz AGOLINOU ${ }^{3}$ \\ et Martin AKOGBETO ${ }^{2,3}$ \\ ${ }^{I}$ Ecole Normale Supérieure de Natitingou ; Université Nationale des Sciences, Technologies, Ingénierie et \\ Mathématiques (UNSTIM) d'Abomey, Bénin. \\ ${ }^{2}$ Centre de Recherche Entomologique de Cotonou, Bénin. \\ ${ }^{3}$ Faculté des Sciences et Techniques - Université d'Abomey-Calavi, Bénin. \\ *Corresponding author; E-mail: anges33@yahoo.fr
}

\section{RESUME}

La lutte anti-vectorielle basée sur l'utilisation des rideaux imprégnés (RII) au cours des deux dernières décennies a permis de réduire le fardeau du paludisme dans bon nombre de pays africains. A l'heure actuelle où Anopheles gambiae, principal vecteur du paludisme en Afrique a développé une résistance vis-à-vis des insecticides recommandés par l'Organisation Mondiale de la Santé (OMS), est-il opportun d'introduire ces RIIs dans les pays endémiques du paludisme comme le Bénin? C'est pour répondre à cette question que le présent travail a été réalisé pour évaluer l'efficacité des RIIs qui seront destinés aux populations. Dans un premier temps, les RIIs ont été soumis au test en cône et en tunnel suivant le protocole de l'OMS où 24 heures après, les taux de mortalité de la souche sensible de An. gambiae kisumu et de la population sauvage en provenance de Ladji ont été comparés à la valeur standard de l'OMS. Dans un deuxième temps, nous avons évalué l'efficacité des RIIs en fonction des savons et des fréquences de lavage. Il ressort des travaux que les résultats des bioessais des tests en cône ont montré que la mortalité induite après un contact de 3 min est de $48,85 \%$ avec la souche sensible Kisumu et $6,96 \%$ pour la population sauvage Ladji. Aussi, l'efficacité des rideaux a donné $65,5 \%$ de taux de mortalité avec la souche de Kisumu et $40 \%$ pour la population sauvage de terrain Ladji. Tous les savons utilisés (klin, fanico, palmida, kogui et koto) ont provoqué une baisse d'efficacité des RIIs à partir du $1^{\text {er }}$ lavage. Des résultats obtenus de cette étude, il ressort que les rideaux imprégnés à la bifenthrine ont été peu efficaces comme moyen de lutte contre le paludisme. Dans un contexte de résistance des vecteurs du paludisme aux insecticides, l'introduction de rideaux imprégnés aux pyrethrinoïdes ne jouerait aucun rôle de barrière chimique pour limiter ou réduire la transmission du paludisme. Il serait souhaitable d'éviter la vulgarisation de tel outil dans la lutte contre le paludisme.

(C) 2018 International Formulae Group. All rights reserved.

Mots clés : Rideaux imprégnés, Anopheles gambiae, efficacité, savon, Bénin. 


\title{
Quality control of curtains impregnated with bifenthrin for wider acceptance and use in Benin
}

\begin{abstract}
Over the past two decades, vector control strategy based on the use of Impregnated curtains (ICs) has helped to reduce the burden of malaria in many African countries. Actually when Anopheles gambiae, the main malaria vector in Africa, has developed resistance against first-line insecticides recommended by the World Health Organization (WHO), is it appropriate to introduce these ICs in endemic countries such as Benin? To answer this question, the present study has been conducted to assess the efficacy of ICs that will be intended for the populations. Ics impregnated with bifenthrin were subjected to standard WHO cone bioassays and tunnel tests.Twenty-four hours after exposure, the mortality rates of the susceptible strain of Anopheles gambiae Kisumu and the wild-resistant strain from Ladji (Benin) were compared with the standard WHO values. Finally, we evaluated the efficacy of ICs in terms of washing practices including types of soaps and washing frequencies. It appears from this work that in cone bioassays, the mortality induced after 3 minutes of exposure to ICs was $48.85 \%$ for the susceptible strain Kisumu and $6.96 \%$ for the wild strain Ladji. The $24 \mathrm{~h}$ mortality rates after exposure to ICs were $65.5 \%$ for the susceptible strain and $40 \%$ for the wild strain. All the soaps used (klin, fanico, palmida, kogui and koto) caused a decrease in the efficacy of the Ics after the first wash. Based on the results obtained in this study, it is apparent that curtains impregnated with bifenthrin remain little effective against malaria vectors. In a context of resistance of malaria vectors to insecticides, the introduction of pyrethroids-impregnated curtains might not play a major chemical barrier role to limit or reduce malaria transmission. It would be desirable to avoid the dissemination of ICs as vector control tools to fight against malaria.
\end{abstract}

(C) 2018 International Formulae Group. All rights reserved.

Keywords: impregnated curtains, Anopheles gambiae, efficacy, washing practices, Benin.

\section{INTRODUCTION}

Première affection parasitaire mondiale, le paludisme constitue aujourd'hui un réel problème de santé publique qui menace plus d'un milliard de personnes situées dans la ceinture de la pauvreté (WHO, 2010). Il demeure l'un des fléaux majeurs des pays les plus pauvres de l'Amérique du Sud à l'Asie du Sud-Est, en passant par l'Afrique subsaharienne où surviennent $90 \%$ des décès dus à la maladie notamment chez les enfants de moins de cinq ans et les femmes enceintes (WHO, 2010).

Au Bénin, il demeure la première cause de fréquentation dans les formations sanitaires avec une fréquence de $37 \%$ chez les adultes et $41 \%$ chez les enfants de moins de 5 ans (Akogbeto et al., 2010). Le plan stratégique national de lutte contre cette maladie dans la plupart des pays en Afrique au Sud du Sahara repose sur 3 composantes majeures à savoir : i) le traitement curatif précoce par les Combinaisons Thérapeutiques à base d'Artémisinine (CTA) ; ii) le Traitement Préventif Intermittent (TPI) par la
Sulfadoxine-Pyriméthamine chez la femme enceinte ; iii) la lutte anti-vectorielle. Cette dernière est basée sur l'utilisation des Moustiquaires Imprégnées d'Insecticide à Longue Durée d'action (MIILD), la Pulvérisation Intra-domiciliaire (PID) d'insecticide et l'épandage des larvicides biologiques (Kelly-Hope L et al., 2008). La lutte anti-vectorielle par l'utilisation des Matériaux Imprégnés d'Insecticide (MII) a permis d'éliminer le paludisme dans sept pays (Emirats Arabe Unis ; Maroc ; Turkménistan ; Arménie; Maldives; Sri Lanka et Kyrgyzstan) et de réduire la prévalence palustre dans certains pays comme la République Démocratique du Congo, le Bénin, le Mali et le Sénégal (WHO, 2016).

Au Bénin, le principal moyen de lutte anti-vectorielle repose essentiellement sur l'utilisation des MILDs. Beaucoup d'efforts ont été consentis depuis la dernière décennie dans bon nombre de pays africains pour augmenter l'accessibilité des populations aux moustiquaires imprégnées, en particulier aux enfants de moins de cinq ans et aux femmes 
enceintes. En effet, l'utilisation de ces Matériaux Imprégnés d'Insecticide (MII) a montré qu'on pouvait réduire la morbidité palustre de 50 à $60 \%$ et la mortalité générale de $20 \%$ en Afrique (WHO, 2016). Malheureusement, des problèmes importants subsistent mettant en danger les objectifs et la pérennité des réalisations. La résistance des vecteurs $\mathrm{du}$ paludisme aux insecticides constitue un handicap à l'utilisation des matériaux imprégnés.

Suite à la baisse observée de sensibilité de An. gambiae aux pyréthrinoïdes, la résistance des vecteurs aux insecticides est devenue un domaine de recherche prioritaire en Afrique depuis quelques années (Aïkpon. et al., 2013; Yadouleton et al., 2011). En plus du problème de résistance, il est à noter que bon nombre de fabricants ne respectent pas les critères de maillage et de durabilité des RIIs, ce qui impacte sur l'efficacité des RIIs.

Devant cette situation, la réintroduction des RIIs comme moyen de lutte anti-vectorielle utilisé dans les années 1990 au Burkina-Faso pourraient-ils être considérés comme outil efficace pour réduire la transmission du paludisme ? C'est dans cette optique que certains pays africains dont le Bénin, a décidé d'avoir recours à des RIIs comme moyen de lutte anti- vectorielle pour lutter contre le paludisme.

Cependant, l'introduction et la vulgarisation des RIIs nécessitent des études préalables au laboratoire suivant les recommandations de l'OMS (WHO, 1998). Cette étude est nécessaire car le développement de la résistance des moustiques aux insecticides, associé à une mauvaise qualité de l'imprégnation constitueront de façon évidente un véritable frein à l'efficacité des RIIs. En plus de ces recommandations de l'OMS, la spécificité de cette étude est l'évaluation des RIIs en milieu communautaire en rapport avec les fréquences de lavage aux différents types de savons lesquels sont fréquemment utilisés par les populations.

Le présent sujet a été donc initié pour vérifier l'efficacité des RIIs au laboratoire et en condition de terrain pour leur utilisation à grande échelle par la communauté dans la lutte contre la transmission du paludisme.
MATERIEL ET METHODES

\section{Evaluation de l'efficacité des rideaux} imprégnés d'insecticide

Les rideaux utilisés dans cette étude avaient été imprégnés à la bifenthrine, un insecticide appartenant à la famille des pyréthrinoïdes. Pour l'evalutation, les RIIs avaient été soumis à deux tests entomologiques à savoir : le test en cône et le test en tunnel. En plus de ces deux tests, le test en cylindre avait été réalisé en considérant ces RIIs comme des papiers imprégnés d'insecticide. Ceci est une recommandation de l'OMS lorsque la dose du matériel imprégné n'est pas connue.

Lesdits tests ont été réalisés avec la souche sensible de référence Kisumu de Anopheles gambiae et une population sauvage de terrain (Ladji) de Anopheles gambiae. Le choix de la population sauvage de terrain Ladji a été fait dans un but unique de comparaison.

\section{Test en cône}

Le test en cône a été conduit suivant le protocole de l'OMS (WHO, 1998). Quatre cônes ont été placés sur les diverses faces de chaque rideau à tester. Au niveau de chaque cône, huit jeunes femelles (2-5 jours) de Anopheles gambiae Kisumu ont été introduites et laissées en contact des rideaux pendant 3 minutes puis retirées des cônes et transférés dans des gobelets stériles voilés sur lesquels ont été posés des tampons de coton imbibés de jus sucré (solution de sucre à 10\%) qui servent à nourrir les moustiques. Parallèlement, les populations sauvages de Anopheles gambiae en provenance de Ladji ont été aussi mis en contact des RIIs suivant le même schéma que la souche Kisumu décrite plus haut.

Après 24 heures de mise en observation, les taux de mortalité ont été enregistrés au niveau de chaque lot de moustiques (souche sensible et population sauvage).

\section{Test en tunnel}

Ce test permet d'évaluer la présence de l'insecticide sur les supports imprégnés. Il est utilisé en complément du test en cône afin 
d'avoir des informations additionnelles sur l'efficacité des rideaux imprégnés.

Cent femelles d'Anopheles gambiae Kisumu (5-8 jours) ont été transférées dans un tunnel au bout duquel il y avait un appât sur lequel les moustiques avaient été nourris. Avant d'atteindre l'appât, les moustiques doivent traverser des fragments de rideaux imprégnés et qui avaient été découpés en carré de $20 \mathrm{~cm}$ de côté et perforés. Les taux de mortalité liés au contact libre des moustiques utilisés avec les rideaux imprégnés ont été déterminés après 15 heures d'exposition des femelles d'anophèles dans le tunnel.

\section{Tests en cylindre}

Les larves de An. gambiae en provenance de Ladji et récoltées ont été élevées à l'insectarium du CREC et les femelles âgées de 2 à 5 jours ont été choisies pour les tests de sensibilité. Les tests ont été réalisés à partir des fragments de rideaux imprégnés à la bifenthrine et découpés en fragment de $5 \mathrm{~cm}$ sur $5 \mathrm{~cm}$ et utilisés comme des papiers imprégnés d'insecticide. Les tests ont été réalisés suivant le protocole OMS en tube cylindrique (WHO, 1998). Le temps d'exposition des moustiques aux rideaux imprégnés est de 60 minutes et le temps d'observation avant la lecture des résultats a été de 24 heures. Dès exposition des moustiques aux RIIs, le nombre de moustiques "knocked-down" $(k d)$, c'est-à-dire qui étaient tombés inanimés au fond des tubes OMS a été noté après $10,15,20,30,45,60$ minutes. Après les tests, les moustiques morts et vivants ont été conservés séparément sur du silicagel dans des tubes eppendorf et stockés à $-20{ }^{\circ} \mathrm{C}$ pour la recherche des mécanismes de résistance.

En tenant compte de l'échelle de l'OMS, toute population de An. gambiae a été considérée comme sensible à l'insecticide, lorsque la mortalité au test a été supérieure ou égale à 97\%. Lorsque la mortalité est inférieure à $80 \%$ la population a été qualifiée de résistante. Entre les deux valeurs, la résistance a été soupçonnée.
Impact des fréquences de lavage et des détergents sur l'efficacité des rideaux imprégnés

Dans le but de connaître l'impact des fréquences de lavage et des détergents sur l'efficacité des RIIS, cinq différents savons régulièrement utilisés par les populations ont été utilisés pour le test. Les fragments de rideaux ont été soumis aux tests en cône et en tunnel suivant le protocole précédemment décrit après $1 ; 3$ et 5 lavages.

\section{RESULTATS}

\section{Evaluation de l'efficacité des rideaux imprégnés \\ Test en cône}

Plus de 100 moustiques ont été utilisés pour effectuer ce test. Les résultats des bioessais après quatre répétitions des tests en cône ont montré que la moyenne du taux de mortalité induite après un contact de 3 minutes a été de $48,85 \%$ avec la souche sensible Kisumu et $6,96 \%$ pour la population sauvage de terrain (Tableau 1).

En se basant sur l'échelle de l'OMS qui indique une valeur $\geq 80 \%$ pour l'acceptabilité du matériel imprégné avec la souche sensible, le taux de mortalité obtenu avec la souche Kisumu (48,85\%) en contact des RIIs reste largement en dessous de la norme requise $(\mathrm{P}<0,05)$.

\section{Test en tunnel}

$\mathrm{Au}$ total, 400 moustiques ont été utilisés pour la réalisation de ce test. Les résultats (Tableau 2) des bioessais après quatre répétitions des tests ont montré que la moyenne du taux de mortalité a été de 65,5\% avec la souche de Kisumu et $40 \%$ pour la population sauvage de terrain Ladji.

\section{Test en cylindre}

Les populations de An. gambiae s.l. qui ont été mises en contact des fragments de RIIs ont développé une forte résistance vis-à-vis de la bifenhtrine, insecticide utilisé pour l'imprégnation des rideaux. Les taux de mortalité obtenus $24 \mathrm{~h}$ après ont été de $16,83 \%$ et de $7,1 \%$ respectivement pour la souche Kisumu et la population sauvage de Ladji (Tableau 3).

En se basant sur l'échelle de l'OMS pour l'interprétation des taux de mortalités, 
ces divers taux obtenus ont été largement en dessous de $90 \%(\mathrm{p}<0,05)$.

\section{Impacts des pratiques de lavage sur \\ l'efficacité des rideaux imprégnés à la bifentrhine}

Tous les savons utilisés (klin, fanico, palmida et kogui et koto) ont provoqué une baisse d'efficacité des moustiquaires à partir du premier lavage. Cette baisse d'efficacité a été significative avec les savons koto et kogui par rapport aux autres savons. Ces savons ont provoqué l'accélération de la dégradation de l'insecticide contenu dans les fibres de coton. Le tableau 4 montre pour les tests en cône :

- une diminution du taux de mortalité de la souche kisumu (40\% à $22 \%)$ au premier lavage du savon fanico au savon kogui. Cette diminution s'est accentuée au fur et à mesure que la fréquence de lavage a évolué dans le temps.

- Le même constat a été fait avec la population sauvage où à ce niveau, la diminution a été beaucoup plus prononcée $(5,2 \%$ à $3,8 \%)$ du savon fanico au savon kogui au premier lavage.

- A partir du $3^{\text {ème }}$ lavage jusqu'au 5 ème, tous les moustiques de la population résistante aux pyréthrinoïdes ont été retrouvés vivants quel que soit le savon.

- Le même constat a été fait avec les tests en tunnel (Tableau 4).

Tableau 1: Mortalité des populations de Anopheles gambiae observée après 3 minutes de contact avec les rideaux imprégnés à la bifenthrine.

\begin{tabular}{ccc}
\hline Répétition & Mortalité (\%) après 3 min de contact \\
\hline & $\begin{array}{c}\text { Kisumu } \\
\text { (souche sensible) }\end{array}$ & $\begin{array}{c}\text { Ladji } \\
\text { (Population sauvage) }\end{array}$ \\
\hline Rep 1 & 48,7 & 7,2 \\
Rep 2 & 49 & 6,9 \\
Rep 3 & 49,2 & 6,85 \\
Rep 4 & 48,5 & 6,92 \\
Moyenne & 48,85 & 6,96 \\
\hline
\end{tabular}

Tableau 2 : Résultat des tests en tunnel après 15 heures de contact des populations de Anopheles gambiae s.l. avec les rideaux imprégnés.

\begin{tabular}{ccc}
\hline Répétition & \multicolumn{2}{c}{$\begin{array}{c}\text { Mortalité (\%) après } \\
\text { de contact }\end{array}$} \\
\hline & $\begin{array}{c}\text { Kisumu } \\
\text { (souche sensible) }\end{array}$ & $\begin{array}{c}\text { Ladji } \\
\text { (Population sauvage) }\end{array}$ \\
\hline Rep 1 & 65 & 39 \\
Rep 2 & 67 & 37 \\
Rep 3 & 64 & 38 \\
Rep 4 & 66 & 38,5 \\
Moyenne & 65,5 & 38,1 \\
\hline
\end{tabular}


Tableau 3 : Taux de mortalité des populations de Anopheles gambiae s.l issus du test en cylindre

\begin{tabular}{ccc}
\hline Répétition & Kortalité (\%) après $\mathbf{2 4} \mathbf{~ h}$ \\
\hline & $\begin{array}{c}\text { Kisumu } \\
\text { (souche sensible) }\end{array}$ & $\begin{array}{c}\text { Ladji } \\
\text { (Population sauvage) }\end{array}$ \\
\hline Rep 1 & 18 & 8,1 \\
Rep 2 & 15,85 & 6,4 \\
Rep 3 & 16,1 & 7,2 \\
Rep 4 & 17,4 & 6,8 \\
Moyenne & 16,83 & 7,1 \\
\hline
\end{tabular}

Tableau 4 : résultats des tests en cône et en tunnel en fonction de la nature du détergent et de la fréquence de lavage des rideaux imprégnés.

Test en cône

\% de mortalité

\begin{tabular}{llcccc}
\hline Fréquence de lavage & Types de detergent & Kisumu & Ladji & Kisumu & Ladji \\
\hline 1 & fanico & 40 & 5,30 & 62,3 & 34,8 \\
& palmida & 38,5 & 5,10 & 58,7 & 31,3 \\
& klin & 31,2 & 4,8 & 53,2 & 29,6 \\
& koto & 23,2 & 2,90 & 48,6 & 25,4 \\
& kogui & 22 & 3,80 & 44,1 & 24,2 \\
\hline 3 & fanico & 18,3 & 0 & 34,3 & 0 \\
& palmida & 16,2 & 0 & 30,1 & 0 \\
& klin & 12,4 & 0 & 23,4 & 0 \\
& koto & 7,3 & 0 & 13,1 & 0 \\
& kogui & 6,5 & 0 & 10,2 & 0 \\
\hline 5 & fanico & 7,1 & 0 & 12,1 & 0 \\
& palmida & 5,2 & 0 & 8,2 & 0 \\
klin & 0 & 0 & 3,1 & 0 \\
koto & 0 & 0 & 0 & 0 \\
kogui & 0 & 0 & 0 & 0 \\
\hline & & & &
\end{tabular}




\section{DISCUSSION}

Des résultats obtenus de l'évaluation des rideaux suivant les trois protocoles décrits précédemment, il ressort que les RIIs évalués n'étaient efficaces ni sur la population locale des anophèles récoltés à Ladji ni sur la souche sensible.

En effet, les résultats du Tableau 3 montrent, dans l'ensemble, un taux de mortalité inférieur au taux recommandé par l'OMS et ceci déjà au premier lavage quel que soit le type de savon. Le même constat a été fait à zéro lavage (Tableau 1) lors des tests en cône.

Cette situation pourrait être due à une mauvaise imprégnation des rideaux par l'industriel.

Par ailleurs, les résultats des tests en tunnel ont montré un taux de mortalité inférieur à $80 \%$ avant lavage quelle que soit la population de Anopheles gambiae mise en contact des RIIs.

Ces faibles taux de mortalité qui ont été enregistrés lors des tests en cône et en tunnel pour évaluer l'efficacité des RIIs s'expliqueraient par le niveau de résistance élevé des populations sauvages de Anopheles gambiae en provenance de Ladji vis-à-vis des pyréthrinoïdes (PY). En effet, au cours de la dernière décennie, l'émergence de la résistance de An. gambiae aux PY a été signalée dans plusieurs pays Africains (Djenontin et al., 2010 ; Etang et al., 2003 ; Hougard et al., 2003).

Par ailleurs, on s'attendait à ce que les résultats des tests en cône et tunnel avec la souche sensible soient supérieurs à $80 \%$ ce qui, n'a pas été le cas (Tableau 2 et 3). Ces deux constats montrent que non seulement les RIIs évalués ont été mal imprégnés, mais aussi l'insecticide utilisé reste inefficace contre les populations sauvages du fait de la résistance de ces dernières vis-à-vis des PY. Ce résultat est en adéquation avec ceux obtenus par Allossogbe et al. (2018) qui ont montré une inefficacité des RIIs imprégnés aux PY et distribuées au sud du Benin dans les zones où An. gambiae a développé une résistance vis-à-vis des $\mathrm{PY}$.

Face à ces constats, il urge que les RIIs testés inefficaces ne soient pas considérés comme moyen de lutte contre les moustiques pour lutter contre la transmission du paludisme.

\section{Conclusion}

Les faibles taux de mortalités des populations de Anopheles gambiae suite aux tests en cône, tunnel et lavage montrent que les RIIs évalués ont une faible efficacité.

Dans un contexte de résistance des vecteurs du paludisme aux insecticides, l'introduction de rideaux imprégnés aux pyrethrinoïdes ne jouerait aucun rôle de barrière chimique pour limiter ou réduire la transmission $\mathrm{du}$ paludisme. Il serait souhaitable d'éviter la vulgarisation de tel outil dans la lutte contre le paludisme.

\section{CONFLIT D'INTERETS}

Les auteurs déclarent qu'ils n'ont pas de conflit d'intérêts.

\section{CONTRIBUTIONS DES AUTEURS}

$\mathrm{AY}, \mathrm{J}-\mathrm{RK}$ et RA ont participé à l'élaboration du protocole, supervisé sa mise application et la rédaction de l'article. FA, $\mathrm{GH}, \mathrm{RT}$ et $\mathrm{AA}$ ont participé à la collecte des données. MA a supervisé tout le travail et la rédaction du document final.

\section{REMERCIEMENTS}

Les auteurs du présent manuscrit remercient le Directeur de l'Ecole Normale Supérieure de Natitingou pour avoir financé ce travail de recherche. 


\section{REFERENCES}

Aïkpon R, Agossa F, Ossè R, Oussou O, Aïzoun N, Oké-Agbo F, Akogbéto M. 2013. Bendiocarb resistance in Anopheles gambiae s.l. populations from Atacora department in Benin, West Africa: a threat for malaria vector control. Parasit Vectors, 6: 192. DOI: doi.org/10.1186/1756-3305-6-192

Akogbéto MC, Djouaka RF, Kinde-Gazard DA. 2006. Screening of pesticide residues in soil and water samples from agricultural settings. Malaria Journal, 5 : 22. DOI: doi.org/10.1186/1475-2875$5-22$

Akogbéto MC, Djouaka, Noukpo H. 2005. Use of agricultural insecticides in Benin. In Bull Soc Path Exo., 98: 400-405.

Akogbéto M, Padonou GG, Gbénou D, Irish S, Yadouleton A. 2010. Bendiocarb: a potential alternative against pyrethroid resistant Anopheles gambiae in Benin, West Africa in Malaria Journal 9: 204210. DOI: doi.org/10.1186/1475-2875-9204

Allossogbe M, Gnanguenon V, Yovogan B, Akinro B, Anagonou R, Agossa F, Houtoukpe A, Padonou GG, Akogbeto M. 2017. WHO cone bio-assays of classical and new-generation long-lasting insecticidal nets call for innovative insecticides targeting the knock-down resistance mechanism in Benin. Malaria Journal, 16: 70-77. $\quad$ DOI: 10.1186/s12936-017-1727-x

Anderson N, Hobo L. 1993. Sociologie des Sans-abris. Nathan : Paris.

Awolola TS, Brooke BD, Koekemoer LL, Coetzee M. 2003. Absence of the kdr mutation in the molecular ' $M$ ' form suggests different pyrethroid resistance mechanisms in the malaria vector mosquito Anopheles gambiae s.s. Tropical Medicine and International
Health, 8: 420-422. DOI: doi.org/10.1046/j.13653156.2003.01034.x

Coetzee M, Craig M, Le Sueur D. 2000. Distribution of African malaria mosquitoes belonging to the Anopheles gambiae complex. Parasitol. 16: 4-77.

David JP, Ismail HM, Chandor-Proust A, Paine MJ. 2012. Role of cytochrome $\mathrm{P} 450$ s in insecticide resistance: impact on the control of mosquito-borne diseases and use of insecticides on Earth. Philos Trans $R$ Soc Lond B Biol Sci., 2013: 368. DOI: 10.1098/rstb.2012.0429

Dia I, Diop T, Rakotoarivony I, Kengne P, Fontenille D. 2003. Bionomics of Anopheles gambiae Giles, An. arabiensis Patton, An. funestus Giles and An. Nili (Theobald) (Diptera: Culicidae) and transmission of Plasmodium falciparum in a Sudano-Guinean zone (Ngari, Senegal). J Med Entomol., 40. 279-283. DOI: doi.org/10.1603/0022-258541.5.901

Djènontin A, Sahabi B, Moiroux N, Henry MC, Bousari O, Chabi J, Ossè R, Koudénoukpo S, Corbel V, Akogbéto M, Chandre F. 2010. Culicidae diversity, malaria transmission and insecticide resistance alleles in malaria vectors in Ouidah Kpomasse-Tori district from Benin (West Africa): A pre-intervention study. Parasit Vectors, 3: 83. DOI: $10.1186 / 1756-3305-3-83$

Etang J, Manga L, Chandre F, Guillet P, Fondjo E, Mimpfoundi R, Toto JC, Fontenille D. 2003. Insecticide susceptibility status of Anopheles gambiae s.l. (Diptera: Culicidae) in the Republic of Cameroon. J Med Entomol., 40: 491-497.

Fanello C, Petrarca V, Torre D, Santolamazza A, Dolo F, Coulibaly G, Alloueche MA, Curtis CG, ToureYT, Coluzzi M. 2003. 
The pyrethroid knock-down resistance gene in the Anopheles gambiae complex in Mali and further indication of incipient speciation within An. gambiae s.s. Insect Mol Biol , 12: 241-245.

Hougard JM, Duchon S, Darriet F, Zaim M, Rogier C, Guillet P. 2003. Comparative performances, under laboratory conditions, of seven pyrethroid insecticides used for impregnation of mosquito nets. Bulletin of the World Health Organization, 81: 324-333.

Snedecor GW, Cochran WG. 1971. Méthodes Statistiques. Edition Association de Coordination Technique Agricole ; 635649;

WHO. 2016. World Malaria Report. World Health Organzation, Geneva.
Yadouleton AW, Martin T, Padonou G, Chandre F, Alex A, Djogbenou L, Dabiré R, Aïkpon R, Glitoh I, Akogbeto MC. 2011. Cotton pest management strategies on the selection of pyrethroid resistance in Anopheles gambiae populations in northern Benin . Parasites and Vectors, 4: $60 . \quad$ DOI: doi.org/10.1186/1756-3305-4-60

Yadouléton AW, N'Guessan R, Allagbé H, Asidi A, Boko M, Osse R, G. Padonou, Gazard K, Akogbéto M. 2010. The impact of the expansion of urban vegetable farming on malaria transmission in major cities of Benin Parasites \& Vectors. 3: 118. DOI: $10.1186 / 1756-3305-3-118$

Zaim M, Guillet P. 2002. Alternative insecticides: an urgent need. Trends Parasitol., 18: 161-163. 